\title{
Management of asthma: a consensus statement
}

\author{
J O WARNER, M GÖTZ, L I LANDAU, H LEVISON, A D MILNER, S PEDERSEN, \\ AND M SILVERMAN
}

SUMmARY In developing these international guidelines there were several unifying themes in the diagnosis and simple management of childhood asthma. For the purposes of the meeting, asthma was operationally defined as 'episodic wheeze and/or cough in a clinical setting where asthma is likely and other rarer conditions have been excluded'.

In making a diagnosis of asthma, a full history is a prerequisite. Additional tests are only used to support clinical impression and to provide objective evidence for therapeutic recommendations. General features of a multidisciplinary approach include an appreciation of the importance of psychosocial factors, counselling, and education. Drugs should be prescribed in a rational sequence: $\beta_{2}$-stimulants for mild episodic wheeze; sodium cromoglycate for mild to moderate asthma; inhaled steroids for moderate to severe asthma; with xanthines, ipratropium bromide, and oral steroids having their place in more persistent and severe cases.

Children and their parents should be reassured that if asthma is properly controlled there is no reason why the child should not lead a normal and physically active life. The management of asthma is rewarding and return to 'normal' lifestyle is nearly always possible with active participation in sporting activities.

Asthma affects more than $10 \%$ of children with a suggestion that prevalence, severity, and mortality has increased in recent years. At the same time there has been a considerable increase in prescribed treatment and hospital admissions for the disease. This is unnecessary as most patients are easily diagnosed and treated. Yet asthma remains commonly underdiagnosed and under treated.

Increased understanding of the pathology of the condition has allowed us to make more rational decisions about therapeutic schedules. Delivery systems for inhaled preparations have improved considerably to facilitate administration even to small children. This paper represents an international agreed policy which should be of value to paediatricians and general practitioners in providing a sound basis for the diagnosis and management of childhood asthma. It is divided into sections to account for the differences between children of different ages. The protocols are outlined in the text and in simple algorithms. To facilitate a pragmatic approach to the condition, asthma is defined as 'episodic wheeze and/or cough in a clinical setting where asthma is likely and other rarer conditions have been excluded'.
Diagnosis of asthma in children unable to perform lung function tests (fig 1)

The use of the label 'asthma' in older children has important clinical consequences. It implies a syndrome consisting of chronic or recurrent wheezing and/or coughing with recognisable variable airway obstruction responsive to a limited range of effective treatments. In children under 5 years, however, we can be neither so confident that wheezing is equivalent to asthma, nor that there is a homogeneous underlying pathogenesis, even in those children who do not have an alternative diagnosis. Even when we confidently make a diagnosis of asthma, the response to treatment is often unsatisfactory in the youngest age group. A number of these controversial points need to be addressed in considering a scheme for the diagnosis of asthma in this age group.

A variety of synonyms have been used to describe asthma in young children (wheezy bronchitis, wheeze associated respiratory infection, infantile asthma, etc). Behind each is the implication that we can recognise variable intrathoracic airway obstruction by means of the clinical feature of recurrent wheeze. Recurrent night cough, and to a lesser 
1066 Warner, Götz, Landau, Levison, Milner, Pedersen, and Silverman

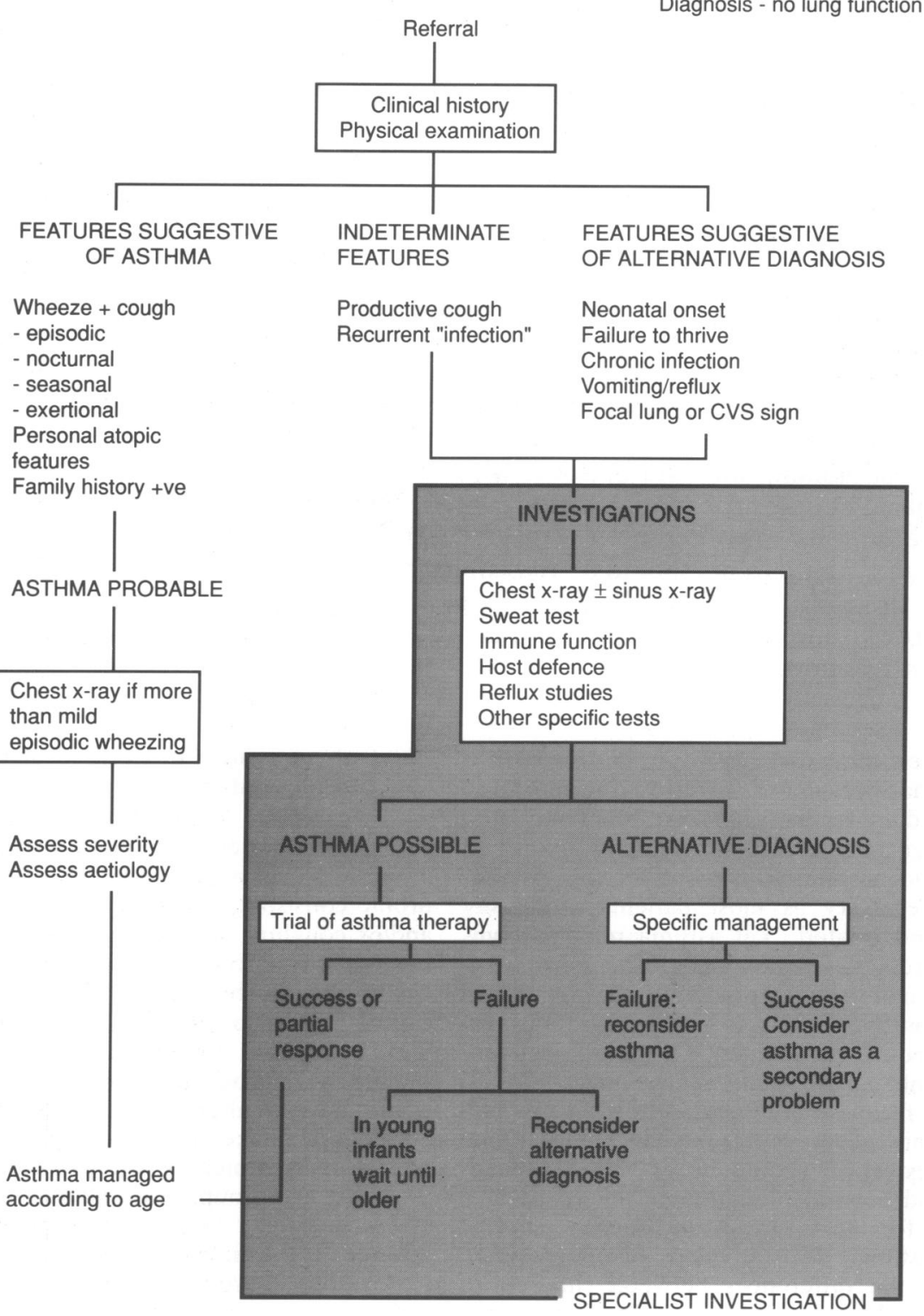

Fig 1 Diagnosis of asthma in children unable to perform lung function tests. CVS=cardiovascular system. 
extent, recurrent spasmodic croup, have also been felt to be features associated with infantile asthma, the implication being that they all result from some degree of irritability of the intrathoracic airways.

There are probably at least two patterns of asthma in infancy. On the one hand there are infants who develop recurrent episodes of wheeze associated with acute viral infections, who come from nonatopic backgrounds, and outgrow their symptoms over the preschool years. On the other hand, there are children with asthma and an atopic background, often associated with eczema and a strong family history, who develop symptoms slightly later in infancy which persist until later in childhood. It may not be possible to distinguish the two patterns of asthma in individual children. However, the term asthma encompasses these extremes.

Asthma is not an all or none diagnosis at any age. There is a range of severity in the population, from entirely normal at one extreme, to severe persistent wheezing at the other. No test, question, or physical sign can provide a precise cut off point. The same limitations apply to the diagnosis of asthma in preschool children.

Wheeze is a feature of intrathoracic airway obstruction. For instance, bronchial stenosis, tracheobronchomalacia, and cystic fibrosis can cause recurrent or persistent wheeze indistinguishable from non-structural, non-focal airways obstruction (that is, asthma). Although wheeze is not synonymous with asthma, asthma is common and alternative diagnoses are rare.

As wheezing in infancy is common, it is quite clear that on occasions children with other diseases (for instance cystic fibrosis or congenital heart disease) will also suffer from asthma. From a practical point of view it is important to recognise that recurrent wheeze in one of these conditions implies the need for a trial of some form of antiasthma treatment such as bronchodilators.

The diagnostic scheme starts with a full clinical history and physical examination. Some features strongly support a diagnosis of asthma, while others are indeterminate or suggestive of alternative diagnoses. There are no definitive diagnostic tests. A chest radiograph should be taken in severe or unusual cases to exclude foreign body and secondary or structural lung disorders such as congenital anomalies. Allergy tests may help in assessing aetiology after a diagnosis has been made. The final diagnostic criterion is a trial of appropriate asthma therapy. When an alternative diagnosis is suspected, (for example, cystic fibrosis, recurrent aspiration, or a disorder of host defences) the patient should be referred to a paediatric respiratory physician for specialist investigation and management.
Diagnosis of asthma in children able to perform lung function tests (fig 2)

The current approach to support the diagnosis is to assess lung function data both at rest and after challenge, and to combine these data with results from allergy tests. A clinical history must be taken with attention to personal, family, and environmental features. A symptom diary is a useful adjunct to clinical history. In most cases, the diagnosis will be obvious at this stage, but lung function data, at rest and after challenge, can be used to support the diagnosis in doubtful cases.

Children from the age of 5-6 years onwards are usually able to perform simple lung function tests such as peak flow rate and forced expiratory volume in one second $\left(F E V_{1}\right)$. More sensitive techniques are generally restricted to specialist centres. Spirometry and flow volume loop measurements, though not essential for a diagnosis of asthma, are useful for differential diagnosis and for the monitoring of patients with severe asthma.

Simple lung function tests can be used to support a diagnosis of asthma in three ways: (1) considerable variability of peak flow rate or $\mathrm{FEV}_{1}$ over short periods of time, for instance during home monitoring. Excessive variability can be defined as a fall of at least $20 \%$ from the established maximum for any individual child. (2) Improvement of at least $20 \%$ in peak flow rate or $\mathrm{FEV}_{1}$ after administration of an inhaled $\beta_{2}$-stimulant. (3) Decrease of $20 \%$ or more in peak flow rate or $\mathrm{FEV}_{1}$ after bronchial challenge. The diagnosis of asthma is hampered in cases of consistently abnormal lung function (that is, no change with bronchodilators or with provocation). In special cases a therapeutic trial of oral steroids may be needed to assist diagnosis.

Bronchial hyper-responsiveness is considered a key feature of asthma. It can be shown in a number of non-asthmatic conditions, however, such as transiently with viral infection, environmental pollution, smoking, atopic dermatitis, and cystic fibrosis. Hyper-responsiveness may also occur in healthy children and conversely asthmatic children may occasionally have no demonstrable hyperresponsiveness.

Exercise is the easiest challenge to perform in the clinic. Of the more sensitive bronchial challenge tests, methacholine is the easiest and least expensive test, whereas histamine is the best standardised. Non-pharmacological challenge tests may either require expensive equipment and maximal patient cooperation (cold air challenge) or are not yet adequately standardised (ultrasonically nebulised distilled water).

The contribution of allergy testing to the diagnosis 


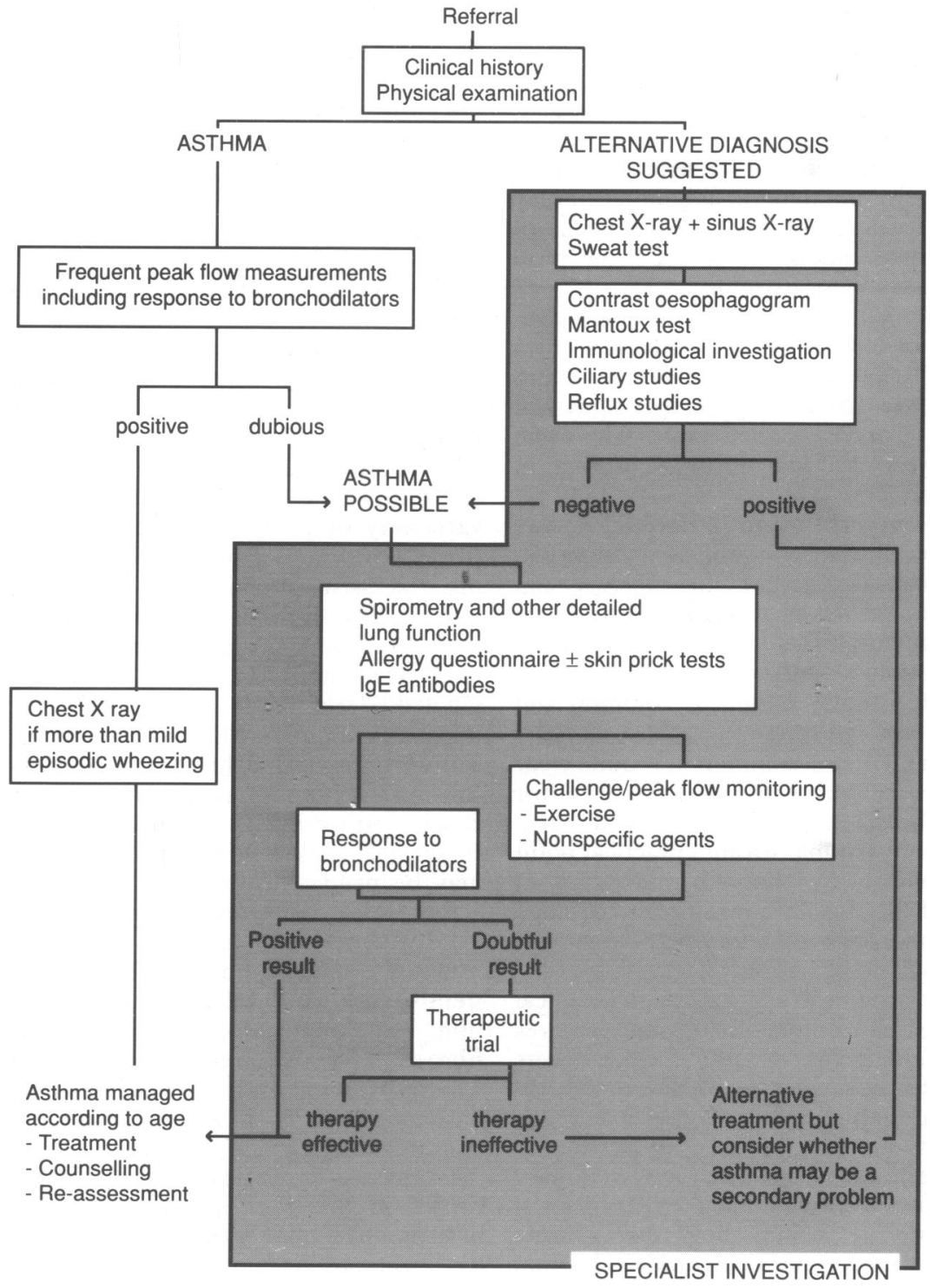

Fig 2 Diagnosis of asthma in children able to perform lung function tests. 
and management of childhood asthma remains controversial. Skin prick tests and/or total IgE concentration may be useful in some children to reinforce a possible diagnosis and indication of causes of asthma. Specific (allergen) bronchial challenges will rarely be required and carry the risk of prolonged late reactions and enhanced bronchial hyper-reactivity. Often the choice of treatment is made irrespective of the results of testing. Nevertheless there are coherent arguments for the determination of offending allergens in order to counsel patients about avoiding specific triggers.

Establishing the diagnosis of asthma is usually straightforward and is supported by lung function studies. However, a therapeutic trial may be necessary to make a final diagnosis.

\section{Common management strategies in children of all ages}

The management goals common to all age groups are to reduce symptoms and optimise lifestyle with few limitations on activity at home, school, work, and leisure, utilising treatments with a low incidence of side effects. This should prevent development of asthma related emotional disorders, avoid hospitalisation, and reduce morbidity and mortality. By the reduction of hyper-responsiveness and the restoration of normal lung function, the prevention of irreversible airway obstruction may be possible.

\section{ENVIRONMENTAL MANIPULATION}

Most children over the age of 5 with asthma have clear evidence of allergy. Precipitating trigger factors should be identified and parents and children counselled on avoidance where possible. There is overwhelming evidence that smoking produces a rapid reduction in lung function. Therefore the avoidance of cigarette smoke must be a priority. House dust mite, the commonest allergen, is difficult to remove but the following measures are helpful: use of plastic mattress covers and pillow cases; removal of feather bedding, bedroom carpet, and soft toys from the bed; and regular vacuum cleaning. If there is convincing evidence of allergy to the family pet it should be removed but if so it will take at least three months for the dander to be totally eliminated from house dust. Exposure to seasonal pollens can be reduced by keeping windows closed during the day and by installing air conditioners and/or air filters, but further work is required to establish their efficacy. In children, asthma is rarely precipitated by food or drink, but if so, avoidance is indicated. Asthma may be exacerbated by emotional disturbance and asthma control can be compromised by adverse psychosocial factors.
Psychotherapeutic management is for the most part simple explanation of the condition but various psychotherapeutic approaches have been shown to be of value as an adjunct to standard drug treatment.

\section{ASSESSMENT OF BASELINE SEVERITY}

Before treating, it is essential to assess lung function at baseline and after full doses of $\beta_{2}$-stimulants to determine what improvements in airflow are possible. Symptom severity should be continually reassessed during treatment. Are the symptoms truly intermittent or do they regularly interfere with sleep, sport, or other activities? Progress can be monitored by diary card record of symptoms. Measurements of peak flow rate should be implemented regularly. Frequency of use of rescue treatments such as $\beta_{2}$-stimulants should be assessed. More sophisticated lung function tests may be necessary for differential diagnosis and for monitoring severe asthma and its management.

\section{SELF MANAGEMENT}

An essential aim for the management of asthma should be to train the child and/or the parents to become an effective first line in the management of symptoms and attacks so that they can cope with most of them without immediate recourse to a doctor, but also to have clear guidelines on when it is necessary to seek medical help. For small children the parents will make all the first line management decisions, but this responsibility will pass more and more to the child as adolescence approaches. They should have ready access to medical advice if long term control is poor and for acute severe attacks. Achieving this management aim necessitates instruction of the patient and parents in the use of drugs and apparatus required for asthma control. The management of asthma should be an example of 'shared caring' in which the child, the parent, and the medical advisors act as a team in managing the asthma. Information, including instructions about medication, is most conveniently given in written form so that it can be referred to from time to time. Benefit is derived from self management programmes or education packages in which children are given the same information as parents, but at an appropriate level. Such a package enables the child to recognise signals and so make decisions about self referral (to parents or doctor), self medication, and the avoidance of precipitating factors. Videos are particularly useful in teaching children. As schools are integrally involved in the daily life of the child, the family doctor should provide teachers with a record card which specifies treatment (regular drugs, prophylactics before games periods, and 
symptomatic relief for troublesome symptoms). Additionally children should carry with them a card which provides an action plan for emergency treatment at home and in the hospital.

\section{PHARMACOTHERAPY}

The management section is divided into four age ranges. However, there are a number of common principles which span these groups. In each case there is a progression through a number of therapeutic regimes from the treatment of mild episodic asthma through to that of chronic, persistent and severe asthma. To a considerable extent, assessment of the severity of asthma is based on the response to treatment. However, mild asthma may be classified as discrete attacks occurring less than once a month, or as more frequent minor wheezes. All are responsive to bronchodilators taken no more frequently than two or three times a week. Moderate asthma can be recognised as discrete attacks occurring no more frequently than once a week, and in which no more than two to three doses (per week) of bronchodilators are required. In such circumstances, sodium cromoglycate prophylaxis would be indicated. More severe attacks tends to be less likely to respond to sodium cromoglycate. Attacks occur more than once a week and response to bronchodilators is sometimes poor. Inhaled corticosteroids are likely to be required in such circumstances with the addition of slow release $\beta_{2}$-stimulants and/or xanthines, particularly for nocturnal episodes.

In general, inhaled treatment at all stages is to be preferred. The dose of inhaled $\beta_{2}$-stimulants could be higher than that generally declared in drug data sheets. Thus if required in severe attacks, doses at least up to that which would be acceptable by the oral route could be inhaled-that is, $2 \mathrm{mg}$ of salbutamol or $2.5 \mathrm{mg}$ of terbutaline even for children as young as 2 years of age. The group felt that at present, apart from sodium cromoglycate, there was no effective alternative so called 'mast cell stabiliser', even though double blind trials of ketotifen have shown a small beneficial effect. The use of ketotifen, however, may be indicated in the treatment of atopic dermatitis and/or rhinitis associated with asthma. The dose of sodium cromoglycate initially should be administered four times daily, but may subsequently be reduced to two to three times daily if control is achieved. With powder inhalers, or nebulised solutions, $20 \mathrm{mg}$ per dose is usually administered, and by metered dose inhalers $10 \mathrm{mg}$ per dose is now preferred. However, $2 \mathrm{mg}$ per dose may be used in countries where a $5 \mathrm{mg}$ per dose actuator is not available. Inhaled corticosteroids must be administered using the minimal dose which adequately controls the disease. Beyond $600 \mu \mathrm{g} / \mathrm{day}$ of either beclomethasone or budesonide there is potential for detectable adrenal and growth suppression. However, it must be remembered that severe uncontrolled asthma can also have a significant suppressive effect on growth. Thus lack of an adequate therapeutic response indicates a need for more aggressive therapy. Lack of response may be defined as a requirement for $\beta_{2}$-stimulants more than two to three times per week.

If required in very severe disease, oral steroids should be given as alternate day treatment wherever possible. As there is not enough evidence that methotrexate or gold are appropriate treatments in childhood asthma, use cannot yet be recommended. There is no benefit in using mucolytics or antibiotics.

Attitudes to the value of xanthines in the management of childhood asthma have changed considerably in recent years. There is increasing concern about side effects which include irritability, insomnia, lack of concentration and poor school performance, headaches, nausea, vomiting, and abdominal pain. Such side effects can occur even when using doses in the acceptable therapeutic range. Interactions with viral infections, antibiotics, and many other drugs can sometimes push conventional doses into the toxic range which might lead to convulsions and even more severe consequences. Thus great care is required in using xanthines and in many parts of the world they have been relegated to very much a second line treatment, and then only in slow release formulations.

It is important to recognise when to reduce treatment. Because asthma is a chronic disease, however, patients should ideally be maintained free of symptoms for at least one year before instituting a trial of dosage reduction, which must be assessed using lung function measurements. A regular review of progress at all stages in the management of the disease is mandatory.

\section{IMMUNOTHERAPY}

The role of immunotherapy remains controversial and the policy adopted in different countries varies. A subcommittee of the European Academy of Allergology and Clinical Immunology recently recommended that immunotherapy should rarely be used in children under 5 years. It should be used only when a clear unavoidable allergic trigger is 0 identified. It is less effective when used in the more severe cases and may exacerbate uncontrolled asthma. As mild disease can be safely, effectively, and inexpensively treated with pharmacotherapy, the place for immunotherapy is restricted. It is most beneficial in patients with seasonal rhinoconjunctivitis with mild asthma. The United Kingdom Com- 
mittee on Safety of Medicine has warned that immunotherapy must be under the direction and supervision of trained specialists for two hours after every subcutaneous injection.

Whereas treatment guidelines can be developed it is important to recognise the need for the individual patient to receive individual treatment. The ideal management is often achieved by a process of trial and error, utilising a rational sequence of therapeutic approaches.

\section{Management in the first year of life (fig 3)}

In the first year of life treatment is often unnecessary in children with mild symptoms. However, those with more troublesome symptoms should be given oral $\beta_{2}$-stimulants and/or oral xanthines. In this age group $\beta_{2}$-stimulants are less likely to produce immediate benefit than in older children. If there is a failure to respond, the child should be referred for specialist assessment. The child can then be given inhaled $\beta_{2}$-stimulants from a metered dose inhaler using a spacer with a face mask system or a nebuliser. Nebulised respiratory solutions may produce a temporary deterioration in lung function which may be important if the baby is critically ill at the time. In this situation, nebulised solutions should be given in conjunction with oxygen, and the child should be observed in hospital to monitor any adverse reactions to treatment. Approximately $40 \%$ of wheezy babies in the first year of life show improvement in lung function with inhaled ipratropium bromide. Again, the nebulised ipratropium bromide could produce a temporary deterioration, lasting up to 10 minutes. This does not occur with aerosol administration from a metered dose inhaler and spacer.

Those babies with intermittent severe and persistent symptoms are more difficult to manage. $\mathrm{Pa}$ tients with persistent symptoms may be treated with sodium cromoglycate administered using a nebuliser. If the condition deteriorates further the use of inhaled steroids is indicated. A beclomethasone suspension is less effective largely because very little of the drug is in particles small enough to reach the lower airways, but a preparation of nebulised budesonide looks more encouraging. Inhaled corticosteroids can be given by a metered dose inhaler and spacer. If response to treatment is still poor and severe breathing difficulties are experienced, the addition of oral steroids, preferably on an alternate morning basis, may be necessary. Patients presenting with intermittent severe asthma can be treated using short courses of oral steroids.

\section{Management in the 1-3 year age group (fig 4)}

In the second and third years of life, those with only occasional symptoms, associated with upper respiratory tract infections, can often be managed with $\beta_{2}$ stimulant syrups or oral xanthines. If the child is having intermittent but quite troublesome symptoms, or if side effects are intolerable on oral treatment he or she should be given inhaled $\beta_{2}$-stimulants, either using a nebuliser or metered dose inhaler with spacer. Those with troublesome symptoms that are not adequately controlled should receive specialist care. The appropriate treatment at this stage is nebulised sodium cromoglycate three times a day to which a $\beta_{2}$-stimulants can be added whenever the child has troublesome coughing or wheezing. Those children who fail to respond to the sodium cromoglycate can be tried on inhaled topical steroids, either as nebulised budesonide suspension or from a metered dose inhaler using a spacer with a face mask. Oral steroids (low, alternate day dosing) are advisable only when other treatments are ineffective.

Infrequent severe asthma symptoms can be controlled with inhaled $\beta_{2}$-stimulants administered using a nebuliser. If response to treatment is poor, short courses (three to seven days) of oral steroids and/or xanthines may be used in addition to the existing treatment.

\section{Management in the 3-5 year age group (fig 5)}

Although some children in the 3-5 year age group with mild intermittent symptoms of asthma may only need oral $\beta_{2}$-stimulants, most will require inhaled $\beta_{2}$-stimulants. Most dry powder inhalers require the child to develop sufficient inspiratory flow rate at least $40 \mathrm{l} /$ minute. Only older, wheeze free preschool children can generate this, and therefore powder inhalders should be reserved only for prophylactic treatment in these children. Once their condition deteriorates, however, their inspiratory flow rate is reduced and they derive less benefit from the powder treatment. In such cases the powder inhaler should be replaced with a metered dose inhaler with spacer or a nebuliser. In some, this treatment will be sufficient. $\beta_{2}$-stimulants may be used when the child is wheezy, as a prophylactic before exercise, or three times daily on a regular basis. If inhaled $\beta_{2}$-stimulants are required more than three to four times/week or symptoms are not controlled completely, continuous prophylactic treatment is required with nebulised, metered dose inhaler with spacer or dry powder sodium cromoglycate. Alternatively, slow release xanthines can be also used. Poor response is an indication for referral 
1072 Warner, Götz, Landau, Levison, Milner, Pedersen, and Silverman

MILD

$<1$ year

(not constitutionally disturbed)

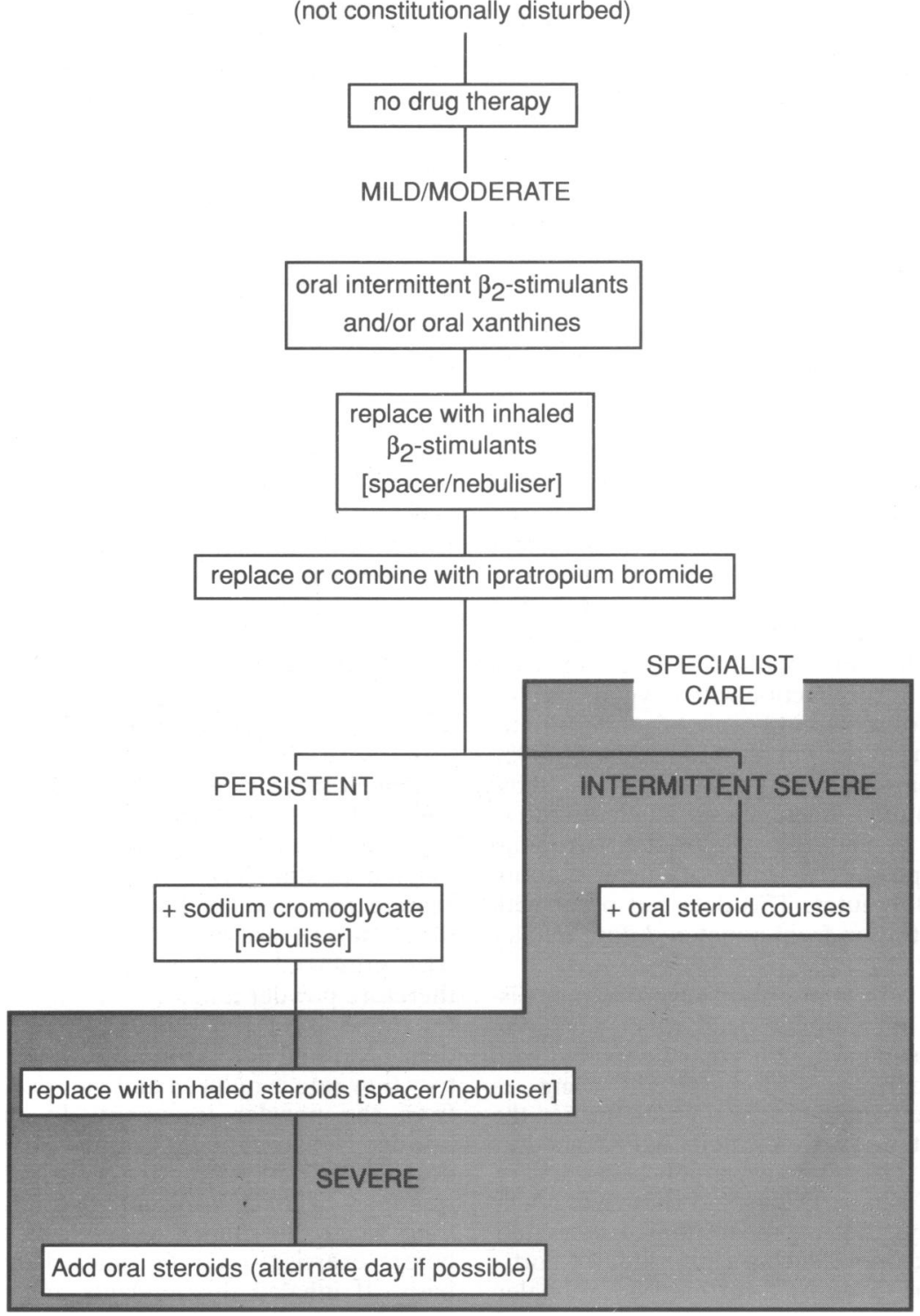

Fig 3 Management in the first year of life. 


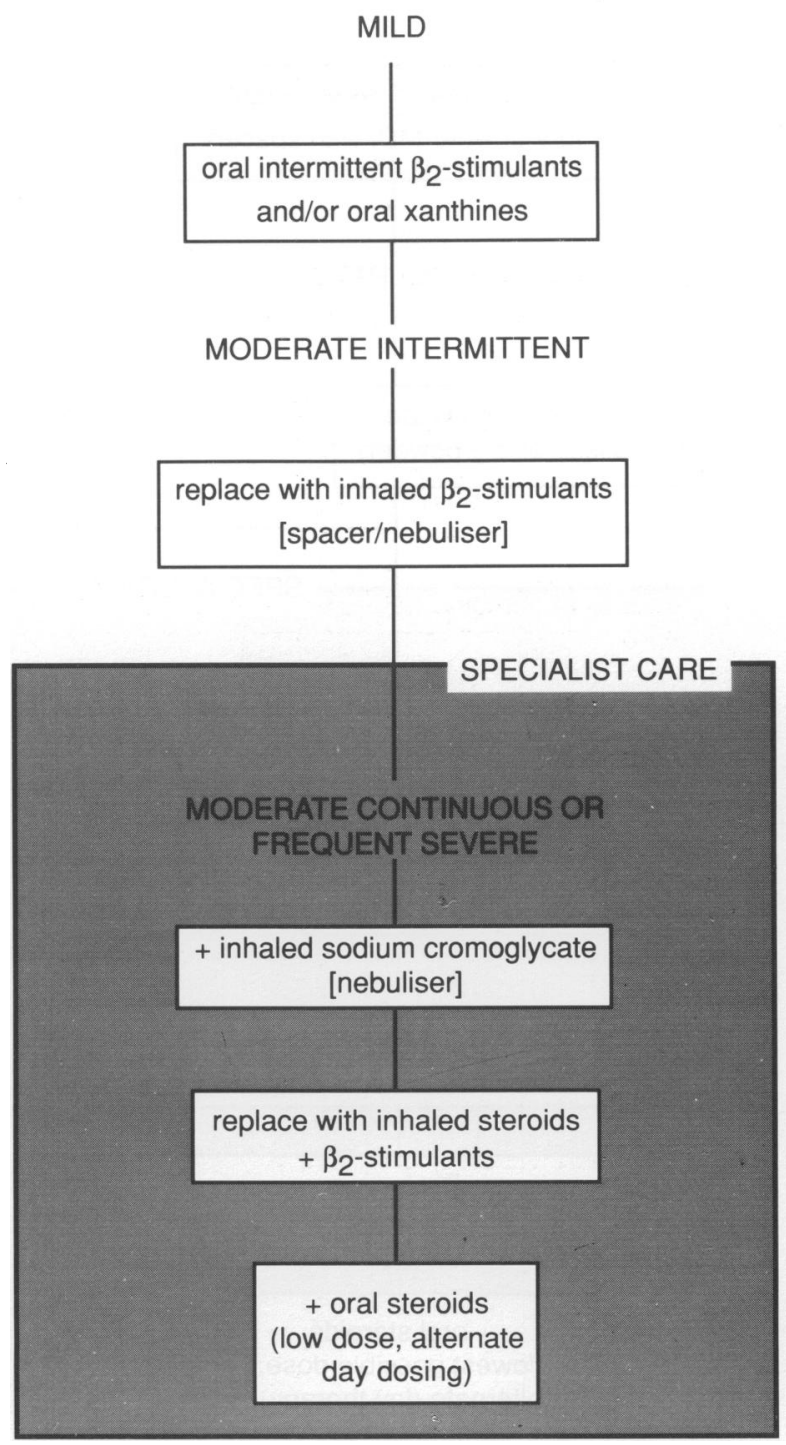

Fig 4 Management in the 1-3 year age group. 


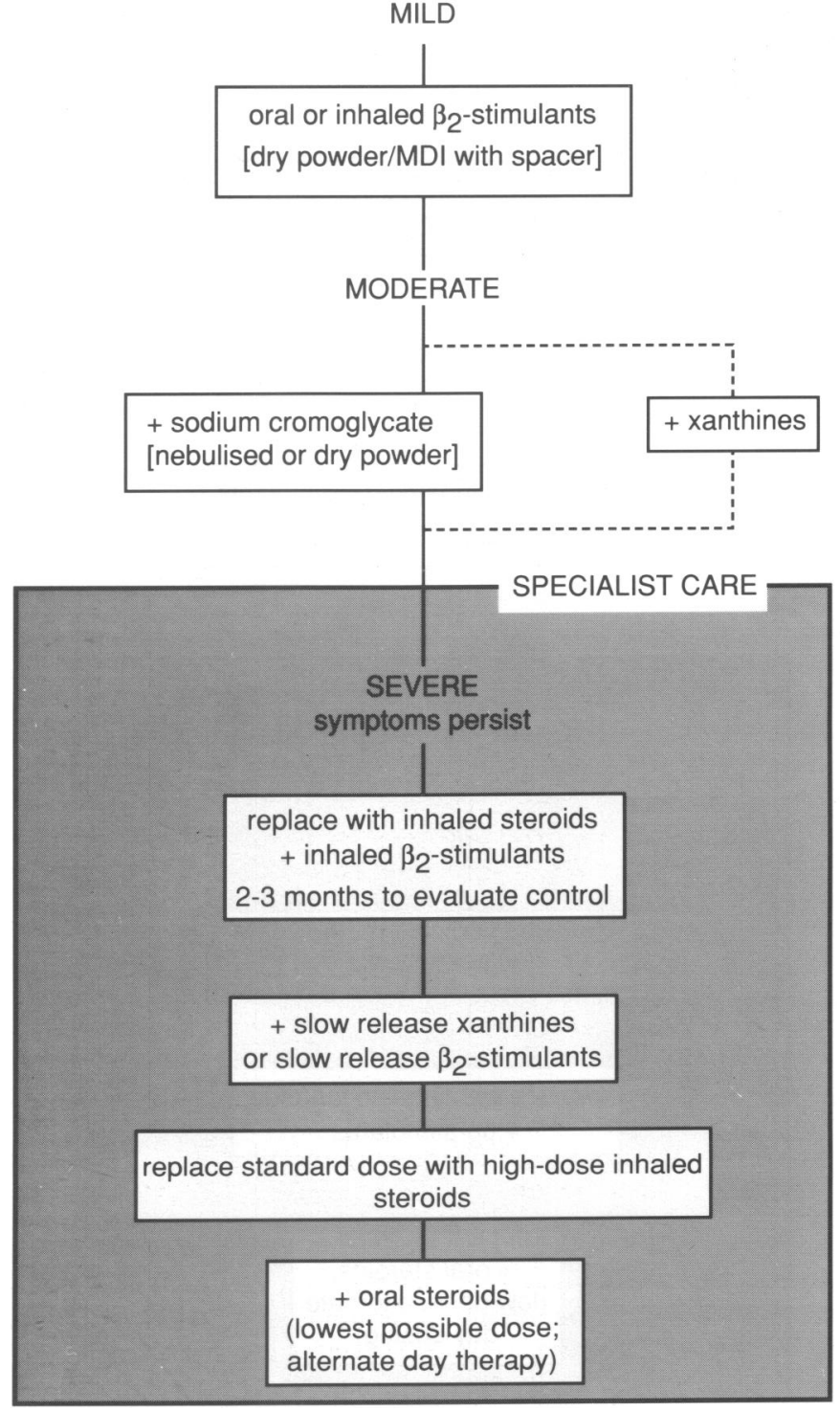

$\overrightarrow{0}$

Fig 5 Management in the 3-5 year age group. MDI=metered dose inhaler. 
to a specialist. Inhaled steroids should be reserved for children with more severe symptoms or for cases where drug administration three to four times daily is not possible. Children, whose symptoms are not completely controlled by sodium cromoglycate and/ or xanthines, may be given a six to eight week course of inhaled steroids to evaluate if they should be switched to this treatment instead.

Initiation of treatment with inhaled steroids is normally at a dose of $200-400 \mu \mathrm{g}$ twice daily for the first one or two weeks. Thereafter, dose adjustments should be made on the basis of clinical response. Although no clinically important side effects have been reported, it is important always to use the lowest dose of inhaled steroid which controls symptoms satisfactorily. Often this can be achieved with a rather low dose once the child's condition is stable.

Slow release xanthines are given in doses sufficient to produce therapeutic blood concentrations (usually equivalent to $16-24 \mathrm{mg} / \mathrm{kg} /$ day of theophylline in two or three doses). When this treatment is prescribed it is important to be aware of possible side effects such as gastrointestinal symptoms, sleep disturbance, poor coordination, and impaired behaviour and learning. Oral $\beta_{2}$-stimulants in a twice daily, slow release formulation may be a suitable alternative.

Continuous treatment with oral steroids should be reserved for the very few cases who cannot be sufficiently controlled by other therapies. The lowest effective dose should be determined and used, and the possibility of alternate day treatment should be explored. However, this regime can normally be replaced by inhaled steroids.

\section{Management in the 5-18 year age group (fig 6)}

Children over 5 years of age can use conventional inhaler devices if initial instruction is thorough. The introduction of dry powder inhalers and spacer devices has improved the success rate particularly in the early school age group (5-14 years).

$\beta_{2}$-stimulants form the first line of treatment for asthmatics in this age group. When used appropriately they can often ensure that the asthmatic child is able to lead an entirely normal life with only minimal symptoms. $\beta_{2}$-stimulants should be administered by inhalation so that the drug is delivered to the bronchial tree for more rapid action. By this route, smaller doses can be used, and adverse effects are minimised. The drugs can be inhaled either from a metered dose inhaler or from an oxygen or compressed air driven nebuliser. The efficiency of metered dose inhalers requires 'hand-lung' coordination, which can be improved by attaching a valved spacer device to the inhaler. Alternatively, a dry powder breath activated system can be used. Oral $\beta_{2}$-stimulants should rarely be used in this age group, although slow release preparations are occasionally considered for nocturnal symptoms.

Sodium cromoglycate should be used when symptoms occur more frequently. In young children, sodium cromoglycate may be given by inhalation as a dry powder. The usual starting dose is $20 \mathrm{mg}$, four times daily. When clinical response occurs, a maintenance dose of $20 \mathrm{mg}$, two or three times daily, may be adequate. A metered dose inhaler, delivering $1 \mathrm{mg}$ per inhalation also has been shown to reduce asthmatic symptoms when given $2 \mathrm{mg}$, four times daily. In many countries a $5 \mathrm{mg}$ metered dose inhaler is available and may be more effective in a dose of $10 \mathrm{mg}$ three to four times a day. Long term use of sodium cromoglycate appears to be safe and virtually free from side effects. If troublesome coughing or wheezing are apparent, an inhaled $\beta_{2^{-}}$ stimulant should be added as required.

Inhaled steroids in the usually recommended doses are the next step of treatment in patients with frequent episodic asthma. Higher dose inhaled steroids can be used to improve overall control of asthma but at an increased risk of side effects, including adrenal and growth suppression.

For severe and persistent symptoms xanthines can be used, although they should be instituted in a stepwise regimen with regular monitoring of blood concentrations. In some countries xanthines would be utilised at an earlier stage. Physicians should also be aware of interactions between xanthines and other drugs. Ipratropium bromide may be added when maximum response with $\beta_{2}$-stimulants can not be achieved without side effects. In non-responders, oral steroids are necessary.

In the adolescent age group (14-18 years) there are several special problems: (1) some teenagers smoke but may not admit this to the family or doctor. The importance of smoking as a trigger factor should be highlighted and the habit aggressively discouraged. (2) Non-compliance and abuse of aerosols is a particular problem and cannister usage must be monitored closely. (3) Most childhood asthma deaths occur in adolescents. (4) Body image is important in adolescents and if asthmatic symptoms are not adequately controlled, the patient may suffer additionally from psychosocial problems. (5) Severe chronic asthma may stunt growth and delay puberty.

The first step in the non-pharmacological management of the adolescent asthmatic is counselling. It is essential to establish rapport. This can be a problem with adolescents and, if so, referral to a colleague may be helpful. Patients should be encouraged to 
1076 Warner, Götz, Landau, Levison, Milner, Pedersen, and Silverman

5-18 years
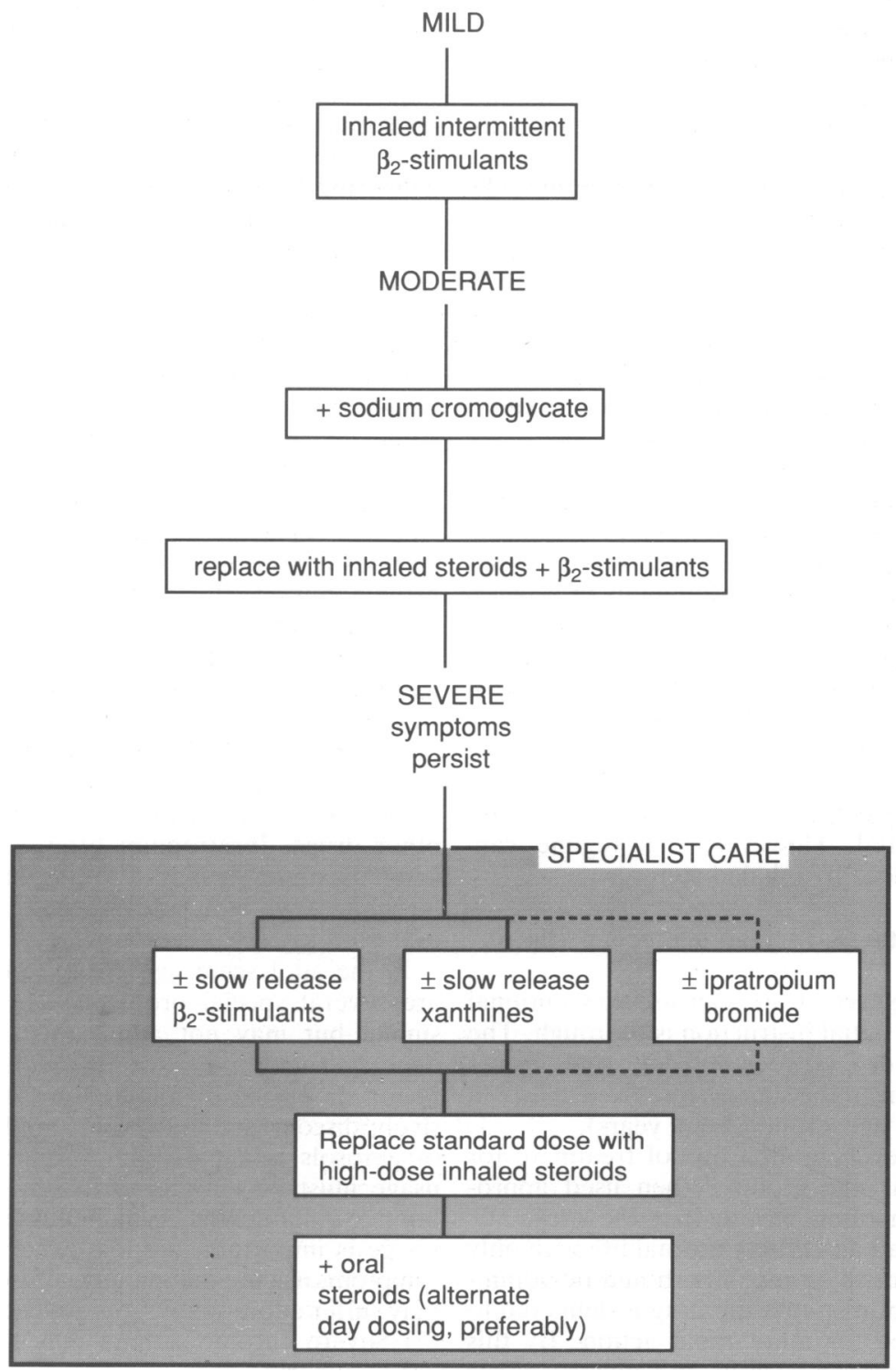

Fig 6 Management in the 5-18 year age group. 
continue to participate in sport but should take prophylatic drugs where necessary and engage in preparatory warm up exercises. Even in adolescents, inhalers are often used incorrectly. Patients should be counselled to seek help when response to their emergency therapy is inadequate. It is important that adolescents do not feel different from their peers. Doctors should be prepared to see such patients out of school hours. Adolescent girls need to be monitored particularly carefully due to the greater likelihood of deterioration. Referral of adolescents to adult clinics should be planned with appropriate introductions and ongoing support. The development of adolescent clinics run jointly by paediatricians and adult chest physicians is to be encouraged.

When symptoms are not relieved by regular treatment, or peak flows are consistently reduced, teenagers should follow an emergency crisis plan. This is perhaps more appropriately referred to as an action plan in order to encourage use before a crisis develops. The action plan, which must be written, usually consists of increasing the doses of routine drugs, and, if necessary, adding oral steroids. A 'fast lane' facility with access to prearranged transport and admission to hospital may be required for those with a risk of severe attacks. Rarely, some will need access to subcutaneous $\beta_{2}$-stimulants or adrenaline and oxygen at home while awaiting transport.

\section{Management of acute severe asthma (fig 7)}

Some patients, particularly in infancy, have infrequent severe attacks of asthma without any signs of asthma between episodes. When such cases have been identified it may be possible to outline and provide a schedule of treatment to handle such episodes at home. Nebuliser and air compressor units can be provided for inhaled $\beta_{2}$-stimulants given up to once every four hours with the addition of a short course of oral steroids (if using prednisolone $1-1.5 \mathrm{mg} / \mathrm{kg}$ daily in one or two doses) until the attack has subsided. Xanthine preparations may also be added to this schedule.

The need for emergency management of acute asthma attacks generally reflects under or delayed treatment which may arise from a failure by parents, patients, and doctors to recognise a deterioration in condition. Acute attacks should have been prevented by adequate prophylaxis and anticipation of problems during stress periods such as acute infection or seasonal high allergen exposure. At such times, an increase in prophylaxis may prevent acute attacks. Once an attack has failed to respond to the standard home treatment, however, medical attention will be required. General practitioners are advised to have an air compressor and nebuliser unit through which a maximal dose of $\beta_{2}$-stimulants can be administered at home. This will not only be therapeutic but will also provide an indication of the need for hospital referral. Failure to respond to the nebulised dose or relapse within four hours of a dose would indicate the need for referral. Response may be gauged on the basis of improvement in peak flow rate and a reduction in respiration rate with improved air entry in the lung fields. Cyanosis in air, the presence of pulsus paradoxus, or peak flow rates of less than $50 \%$ of predicted values after administration of bronchodilator would indicate the need for hospital referral. At this stage it would be appropriate for the general practitioner to give a loading dose of aminophylline by intravenous injection slowly over 20 minutes in a dose of $5 \mathrm{mg} / \mathrm{kg}$. However, if the patient has been receiving an oral theophylline preparation it would be safer to avoid an intravenous theophylline preparation and to use intravenous salbutamol at a dosage of $10 \mathrm{microgram} / \mathrm{kg}$ given slowly. In addition either oral prednisolone $1-2 \mathrm{mg} / \mathrm{kg}$ or intravenous hydrocortisone $4 \mathrm{mg} / \mathrm{kg}$ could be administered.

On arrival at hospital, it is apparent that quite often inappropriate investigations and treatment are initiated. The key parameters to monitor are pulse, colour, the presence of pulsus paradoxus, peak flow rate, arterial blood gases, and/or oxygen saturation. Additional doses of nebulised $\beta_{2}$-stimulants should be administered. If the attack subsides, monitoring should be continued for at least four hours. If the patient remains stable during this period, they can be discharged home on continuing nebulised and oral treatment. If symptoms recur, or are unresponsive to this treatment, hospital admission is necessary.

After admission, the dosage and frequency of $\beta_{2^{-}}$ stimulants by nebuliser can be increased with the mandatory administration of oxygen. At the same time, oral prednisolone $1-2 \mathrm{mg} / \mathrm{kg}$ should be administered. Wheezing induced dehydration should be counteracted by appropriate hydration with the avoidance of over hydration which leads to pulmonary oedema. Under hydration could lead to drying of secretions and electrolyte imbalance which will compromise the effectiveness of treatment. This may necessitate the use of an intravenous infusion. Intravenous aminophylline in a loading dose followed by a continued infusion may also be added at this stage.

Once there is a failure of response to bronchodilators, the patient should be admitted to intensive care, continuing intravenous aminophylline and nebulised $\beta_{2}$-stimulants. Intravenous hydrocortisone $4 \mathrm{mg} / \mathrm{kg}$ every four hours should also be admin- 


\section{Acute management}

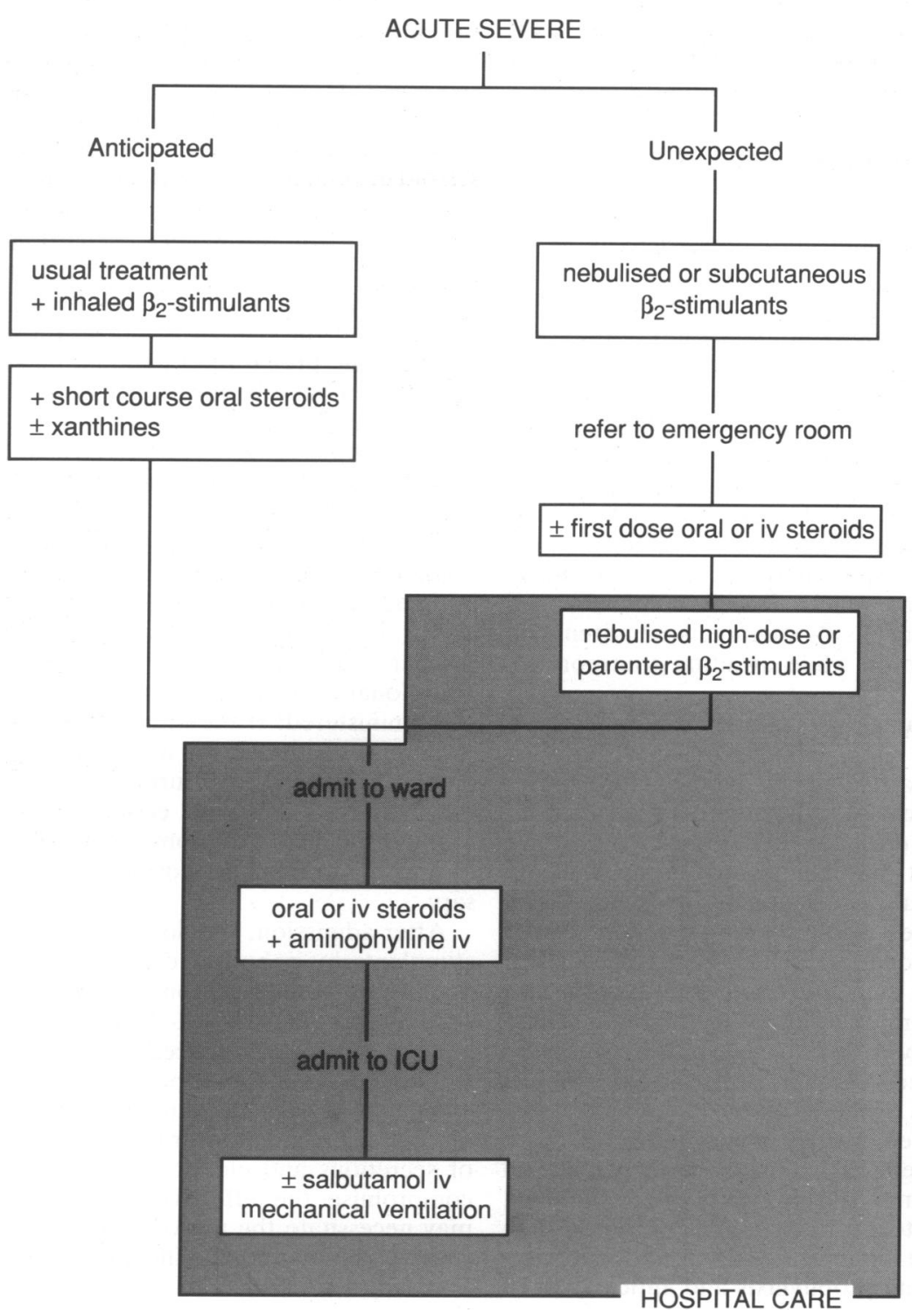

Fig 7 Management of acute severe asthma. ICU=intensive care unit. 
istered. Blood gas monitoring will be required. Hypokalaemia may develop in response to treatment and requires correction. Downes criteria of carbon dioxide pressure $>8.66 \mathrm{kPa}(65 \mathrm{~mm} \mathrm{Hg})$ as an indication when to resort to paralysis/sedation and ventilation may lead to inappropriate and excessive numbers of patients being ventilated.

Finally an acute attack must always be considered a failure of prophylaxis and before discharge the regular treatment should be re-examined.

\section{Direction for future clinical research into management}

During the course of the meeting, a number of areas were highlighted where there was clear need for further clinical research. Long term follow up studies of outcome in various situations are required. The frequency with which asthma is eventually manifested in individuals who are initially detected with asymptomatic bronchial hyperreactivity is not known. Although there is an increasing demand for early introduction of aggressive asthma prophylaxis, the long term outcome of this approach, compared with symptomatic treatment, is unknown. Many individuals who have asthma throughout childhood become asymptomatic in adolescence or early adult life, but retain persistent defects of lung function with measurable lung overinflation. It is not known whether such patients should have continuing aggressive treatment and whether this affects the possibility of recurrence of the disease in later life. Similarly, it is uncertain whether an aggressive approach to the reduction of bronchial hyper-reactivity will improve the long term prognosis. During the discussion on therapy of asthma in the less than 1 year age group, it became apparent that there were very few controlled trials of any form of treatment. In particular, identification of the most appropriate and effective delivery systems for medication is required. The group accepted that immunotherapy had been established to be superior to placebo, but comparisons of this treatment with pharmacotherapy are necessary to evalute its role in the treatment of asthma. The efficacy of environmental manipulations, including the use of filters and ionisers, also requires further investigation. It was agreed that there is a need for new modalities of treatment in very severe oral steroid dependent asthmatics, and trials of the use of methotrexate, azothioprine, cyclosporin, oral gold and troleandomycin are awaited with interest.

This consensus statement was developed at a roundtable meeting (London, 3-4 December 1988) and was accepted by all the participants. Their contributions to the paper are gratefully acknowledged. Steering committee: John Warner, Consultant Paediatric Chest Physician, Brompton Hospital, London, UK; Tony Milner, Department of Child Health, Queens Medical School, Nottingham, UK; Mike Silverman, Paediatric Department, Hammersmith Hospital, London, UK; Manfred Götz, Consultant Paediatrician, Universität-Kinderklink, Wien, Austria; Søren Pedersen, Department of Paediatrics, Kolding Sygehus, Kolding, Denmark; Henry Levison, Chief, Chest Division, Hospital for Sick Children, Toronto, Canada; Louis I Landau, University Department of Paediatrics, Princess Margaret Hospital for Children, Perth, Australia. Participants: V Alzina, Spain; A Andreoli, Italy; A Backman, Finland; K Juntunen-Backman, Finland; KP Dawson, New Zealand; $H$ von der Hardt, Federal Republic of Germany; D Hofmann, Federal Republic of Germany; JG HeurtaLopez, Mexico; Y Iikura, Japan; N-IM Kjellman, Sweden; P König, USA; W Lenney, UK; HJ Neijens, The Netherlands; S Oseid, Norway; J Paupe, France; G Rachelefsky, USA; P Scheinmann, France; R Seger, Switzerland; JE Selcow, USA; MRH Taylor, Ireland.

The group gratefully acknowledges the organisational and editorial skills of Adelphi Communications Limited:

Correspondence to Dr JO Warner, Brompton Hospital, Fulham Road, London SW3 6HP. 